\title{
Molecular dynamics simulations of the electric double layer capacitance of graphene electrodes in monovalent aqueous electrolytes
}

\author{
Gengping Jiang ${ }^{2,3}$, Chi Cheng ${ }^{2,3}$, Dan $\mathrm{Li}^{2,3^{*}}$, and Jefferson Zhe Liu ${ }^{1,3 *}$ \\ ${ }^{1}$ Department of Mechanical and Aerospace Engineering, Monash University, VIC 3800, \\ Australia \\ ${ }^{2}$ Department of Materials Science and Engineering, Monash University, VIC 3800, Australia \\ ${ }^{3}$ Monash Center for Atomically Thin Materials, Monash University, VIC 3800, Australia \\ Correspondence to: zhe.liu@monash.edu; dan.li2@monash.edu
}

\begin{abstract}
Electrical double layer (EDL) capacitors based on recently emergent graphene materials have shown several folds performance improvement compared to conventional porous carbon materials, driving a wave of technology breakthrough in portable and renewable energy storage. Accordingly, much interest has been generated to pursue a comprehensive understanding of the fundamental yet elusive double layer structure at the electrode/electrolyte interface. In this paper, we carried out comprehensive molecular dynamics simulations to obtain a comprehensive picture of how ion type, solvent properties, and charging conditions affect the EDL structure at the graphene electrode surface, and thereby its contribution to capacitance. We show that different symmetrical monovalent aqueous electrolytes $\mathrm{M}^{+} \mathrm{X}^{-}\left(\mathrm{M}^{+}=\mathrm{Na}^{+}, \mathrm{K}^{+}, \mathrm{Ru}^{+}\right.$, and $\mathrm{Cs}^{+} ; \mathrm{X}^{-}=\mathrm{F}^{-}, \mathrm{Cl}^{-}$, and $\left.\mathrm{I}^{-}\right)$indeed have distinctive EDL structures. Larger ions, such as, $\mathrm{Ru}^{+}, \mathrm{Cs}^{+}, \mathrm{Cl}^{-}$, and $\mathrm{I}^{-}$, undergo partial dehydration and penetrate through the first water layer next to the graphene electrode surfaces under charging. As such, the electrical potential distribution through the EDL strongly depends on the ion type. Interestingly, we further reveal that the water can play a critical role in determining the capacitance value. The change of dielectric constant of water in different electrolytes largely cancels out the variance in electric potential drop across the EDL of different ion type. Our simulation sheds new lights on how the interplay between solvent molecules and EDL structure cooperatively contributes to capacitance, which agrees with our experimental results well.
\end{abstract}

Keywords: graphene supercapacitor, molecular dynamics simulations, electric double layer structures, aqueous electrolyte, monovalent ions. 


\section{Introduction}

Electrochemical capacitors, also called supercapacitors, store energy by forming an electric double layer (EDL) of electrolyte ions on conductive porous electrodes, usually made from porous carbon. Recent advance in making graphene-based nanoporous carbon materials have led to much improved capacitive energy storage performance, which directs much research efforts into investigating the fundamental charging mechanism, the formation of EDL at the electrode/electrolyte interface. Indeed, it is not until very recently do we start to understand many of the anomalous phenomena associated with the formation of EDL particularly in small nano-sized pores, for example the drastically increased capacitance and the distortion of ion solvation shell in pores below $2 \mathrm{~nm}$, to name a few [1, 2]. Although the structure and property of EDLs on metal and carbon-based electrode have been widely studied via the various numeric simulation (i.e., continuum modeling [3], classic MD simulation [4-6], grand canonical Monte Carlo [7, 8], and classic density functional theory [9]), many questions remain to be explored such as the effect of ion size, degree of ion hydration, dielectric constant of solvents [10-15], the potential-dependent EDL structure, and the "bell” or "U” shaped differential capacitance $[16,17]$. A comprehensive understanding of the EDL structure and its dependence on various ion type and different charging conditions remains vacant.

Accordingly, in this work, we investigate the molecular structures of EDL in graphene-based electorchemical capacitor systems via molecular dynamic (MD) simulations. Specifically, we are looking at the influence of various ion type and the strength of charging electric field on the structure of EDL. The combination of MD simulations and the aid of Guoy-Chapman-Stern (GCS) model framework [18-21] has allowed a comprehensive understanding on the EDL molecular structure and an in-depth analysis of its effects on the capacitance of graphene-based electrochemical capacitors. Our results show that the ion type indeed plays a crucial role in determining the ion concentration distribution in an EDL. More importantly, our simulations have successfully revealed the significant effect of the dielectric properties of water molecules on the achievable capacitance of a certain aqueous electrolyte.

\section{Methodology}

Figure 1 shows a typical system in our MD simulations: graphene sheets $(10.2 \mathrm{~nm} \times 10.0 \mathrm{~nm})$ aligned in parallel with an interlayer distance of $5 \mathrm{~nm}$. The dashed box depicts the simulation supercell with periodic boundary conditions employed at all three dimensions. Symmetrical 
monovalent aqueous electrolytes $\mathrm{M}^{+} \mathrm{X}^{-}\left(\mathrm{M}^{+}=\mathrm{Na}^{+}, \mathrm{K}^{+}, \mathrm{Ru}^{+}\right.$, and $\mathrm{Cs}^{+} ; \mathrm{X}^{-}=\mathrm{F}^{-}, \mathrm{Cl}^{-}$, and $\left.\mathrm{I}^{-}\right)$were used to fill the gaps between graphene sheets. The concentration was set as $1 \mathrm{~mol} / \mathrm{L}$.

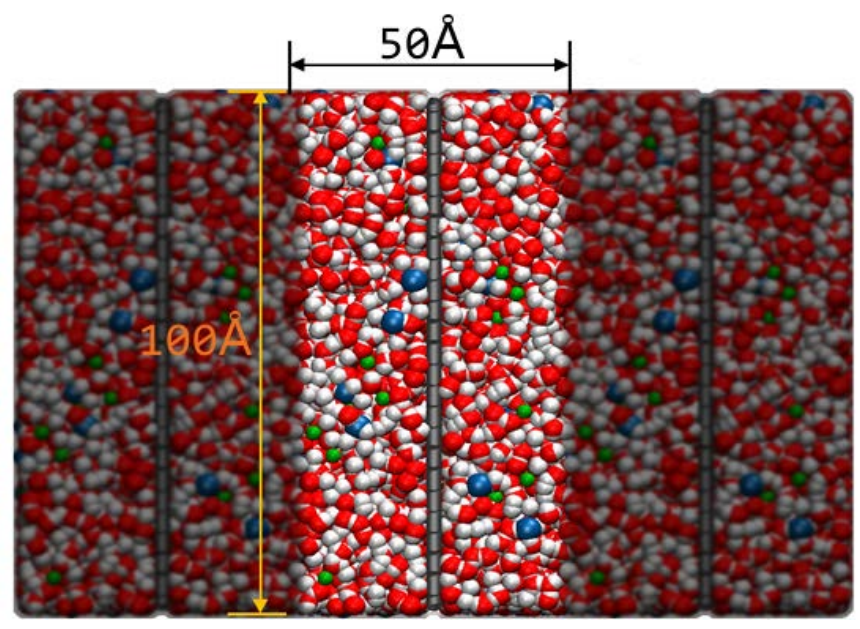

Figure 1. A typical model system employed in the MD simulation to investigate the electric double layer formed at charged graphene sheets immersed in symmetric monovalent electrolyte (1 mol/L).

Given the theoretical intrinsic capacitance value of graphene $(21 \mu \mathrm{F} / \mathrm{cm}$ [22]) and electric potential window for aqueous electrolytes $(1 \mathrm{~V})$, we estimated the maximum charge per carbon atom to be 0.0685 e/C-atom, equivalent to a total charge of 270 electrons in one graphene sheet (3936 carbon atoms) in our system (Fig. 1). Thus a total charge of $0, \pm 60$, or $\pm 160 e^{-}$was imposed on one graphene layer (corresponding to $0, \pm 0.015$, or \pm 0.041 e/C-atom), respectively, so as to consider the influence of different charging conditions on the EDL structure and the resultant capacitance values [6, 23-27]. The injected charges were homogeneous distributed in every carbon atoms across the graphene layer. Some MD simulation techniques have recently been developed to apply a constant electric potential $[28,29]$ on electrodes, which is more consistent with electrochemistry experiment conditions. However, they are computational demanding in comparison with the widely used constant surface charge method. In addition, recent studies showed a negligible difference of simulating the EDL structures between these methods particularly at a low electric potential $(<2 \mathrm{~V})$ [30, 31]. Therefore, in this study, we adopted the conventional constant surface charge method. Water molecules were accordingly filled in the supercell to generate a pressure close to 1 bar at 300 $\mathrm{K}$. The numbers of the electrolyte ions were determined with two conditions: the ion concentration being $1 \mathrm{M}$ and charge neutrality of the whole system. Table 1 summarizes the numbers of water molecules and ions in our MD simulation systems.

Table 1. Number of water molecules and electrolyte ions in one MD simulation supercell under different charging conditions. 


\begin{tabular}{cccc}
\hline $\begin{array}{c}\text { Total charge on one graphene } \\
\text { layer (3936 carbon atoms) }\end{array}$ & $\mathrm{M}^{+}$ & $\mathrm{X}^{-}$ & Water \\
\hline $0 \mathrm{e}$ & 310 & 310 & 48884 \\
$+60 \mathrm{e}$ & 280 & 340 & 48884 \\
$+160 \mathrm{e}$ & 230 & 390 & 48884 \\
$-60 \mathrm{e}$ & 340 & 280 & 48884 \\
$-160 \mathrm{e}$ & 390 & 230 & 48884 \\
\hline
\end{tabular}

The TIP/3P model was used for water because it can best reproduce the dielectric constant of water among other popular water models and the SHAKE algorithm was employed [A1][32, 33]. In addition to the electrostatic forces, the van der Waals interactions among the ions, carbon atoms, and the water molecules were described by using the modified Lenard-Jones potential [34]. Parameters of LJ potentials of most ions and carbon atoms were taken from CHARMM27 force field [35], except those of $\mathrm{I}^{-}$and $\mathrm{F}^{-}$anions that were taken from these references [36, 37]. Table 2 summarizes the parameters and the charges of each type of ions. The Lorentz-Berthelot mixing rule was adopted to determine the LJ potential parameters between different types of ions, waters and carbon atoms.

Table 2. Parameters of the Lennard-Jones force fields that were used in this paper.

\begin{tabular}{ccccc}
\hline Atoms & $\varepsilon(\mathrm{eV})$ & $\sigma(\AA)$ & Molar Mass & Valence (e) \\
\hline $\mathrm{Na}^{+}$ & 0.00203 & 2.430 & 22.990 & -1 \\
$\mathrm{~K}^{+}$ & 0.00377 & 3.143 & 39.102 & +1 \\
$\mathrm{Cs}^{+}$ & 0.00824 & 3.742 & 132.90 & +1 \\
$\mathrm{~F}^{-[37]}$ & 0.00781 & 3.118 & 18.998 & -1 \\
$\mathrm{Cl}^{-}$ & 0.00650 & 4.045 & 35.450 & -1 \\
$\mathrm{I}^{-[36]}$ & 0.00434 & 5.167 & 126.90 & -1 \\
O (TIP3P) & 0.00660 & 3.151 & 15.999 & -0.834 \\
$\mathrm{H}$ (TIP3P) & 0.00000 & 0.000 & 1.0080 & 0.417 \\
$\mathrm{C}$ (graphene) & 0.00304 & 3.550 & 12.011 & $0 \sim \pm 0.041$ \\
\hline
\end{tabular}

Our MD simulations were carried out using the LAMMPS code. Time step was set as 1.5 fs. The van der Waals forces were truncated at $1.0 \mathrm{~nm}$ with long-range Columbic interactions computed using the particle-particle particle-mesh (PPPM) algorithm. Although isolated graphene layers take on a micro-corrugated texture, the corrugation amplitude of the graphene layer [38] is generally much smaller than $5 \mathrm{~nm}$ as selected for this simulation [39, 40]. Furthermore, previous MD studies 
have showed a negligible effect of graphene surface corrugation on the structure of the first water layer, suggesting a minor effect on the EDL [41-43]. As such, we didn't consider the movement of carbon atoms and their positions were frozen. The Berendsen thermostat was adopted to control the system temperature, which was firstly increased from $298 \mathrm{~K}$ to $373 \mathrm{~K}$ over a period of 10ps, maintained at 373K for an additional 10ps before gradually cooled down from 373K back to 298K. Then, the NVT ensemble simulations at $298 \mathrm{~K}$ were carried out for $1.2 \mathrm{~ns}$. Results generated during the last 150ps were used to analyze the EDL structures and to calculate the capacitance. In some cases, longer MD simulations up to 2 ns were carried out to ensure that the systems indeed reached thermal equilibrium state.

We split the channels into a set of bins (of $0.05 \AA$ in width) along the direction ( $x$ ) perpendicular to the graphene surface. The averaged number density of particles (e.g., water, electrolyte ions) within a bin during the last 150ps of our MD simulations was calculated to represent the number densities $\rho$ of the corresponding particles. Such a number density $\rho(x)$ is widely used to represent the EDL structures. The resultant electric potential $V(x)$ can be computed by integrating the following one-dimensional Poisson equation:

$$
\frac{\partial^{2} V(x)}{\partial x^{2}}=-\frac{q(x)}{2 A_{G} \varepsilon_{0}}
$$

where $x$ is the distance to the graphene surface $(x>0), q(x)$ is the total charge quantity at $x, A_{G}$ is the cross-section area of MD simulation, namely the size of graphene sheet, and $\varepsilon_{0}$ is the vacuum dielectric constant. Note that $q(x)$ includes the electrode charge, electrolyte ion charge, and charges of hydrogen and oxygen atoms of water molecules. The term $2 A_{G}$ in Eq. (1) is due to double accounting of electrolyte charges from both sides of graphene surface. We used numerical integration of Eq. (1) to obtain $V(x)$. The $V(x)$ value in the middle of the channel $(x= \pm L / 2)$ was used as a reference. Since the channel is symmetrical, the $d V / d x$ value at $x=L / 2$ should be equal to zero. The integral of Eq. (1) yields the potential $V(x)$.

$$
V(x)=-\iint_{L / 2}^{x} \frac{q(x)}{2 A_{G} \varepsilon_{0}} d x^{2}
$$

\section{Results and Discussions}

\subsection{Structures of the EDL}


We normalized the number densities $\rho$ of ions and water molecules with respect to their number densities in the bulk aqueous solution to obtain the relative number densities $\rho^{*}$. Figure 2 summarizes the results of the electrolyte ions $\mathrm{Na}^{+}, \mathrm{K}^{+}, \mathrm{Rb}^{+}, \mathrm{Cs}^{+}$cation and co-ion $\mathrm{Cl}^{-}$in different aqueous electrolyte close to the negatively charged graphene surfaces. Figure 3 shows the results for $\mathrm{F}^{-}, \mathrm{Cl}^{-}, \mathrm{I}^{-}$anions and counter-ion $\mathrm{Na}^{+}$close to the positively charged graphene surfaces. Figure 4 shows the results for water molecule close to graphene surfaces with either positive or negative charges.

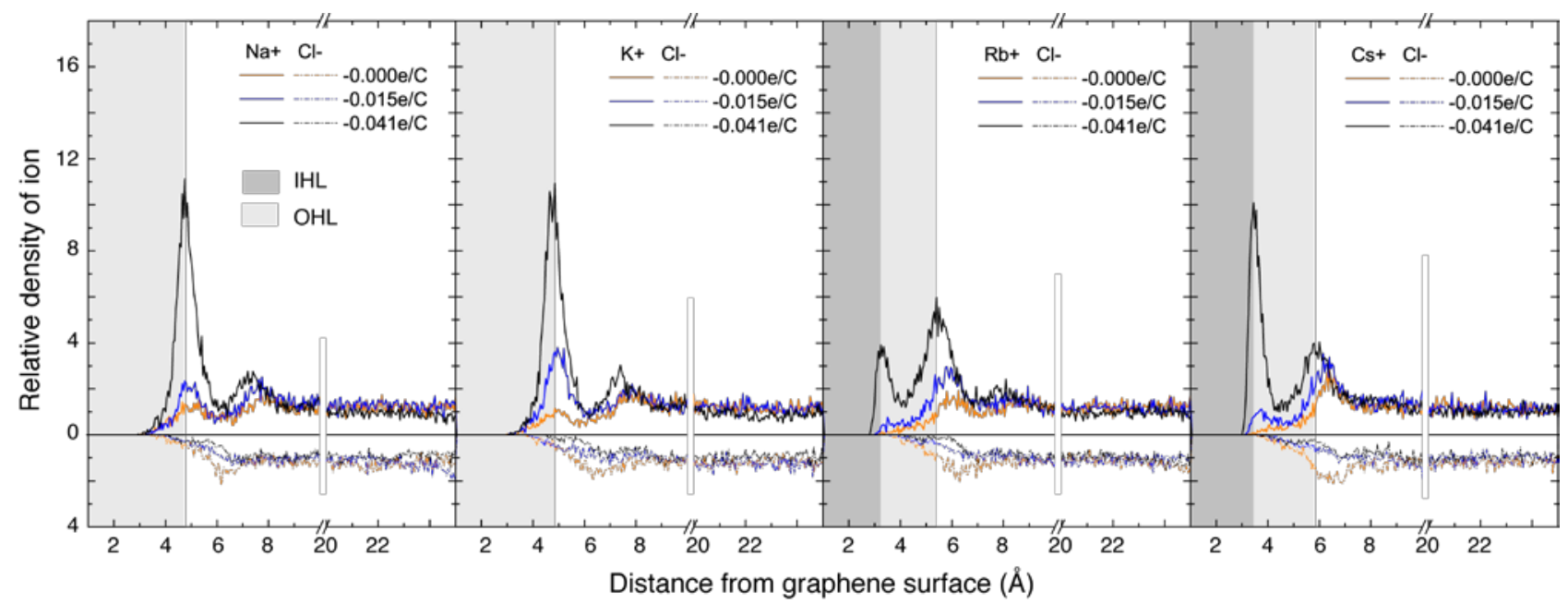

Figure 2. Relative number density $\rho^{*}$ of different electrolyte ions as a function of distance from the graphene cathode surface with a charge of $0,-0.015$ and -0.041 e/C-atom, respectively. The shaded regions represent the inner Helmholtz layer (IHL) and outer Helmholtz layer (OHL).

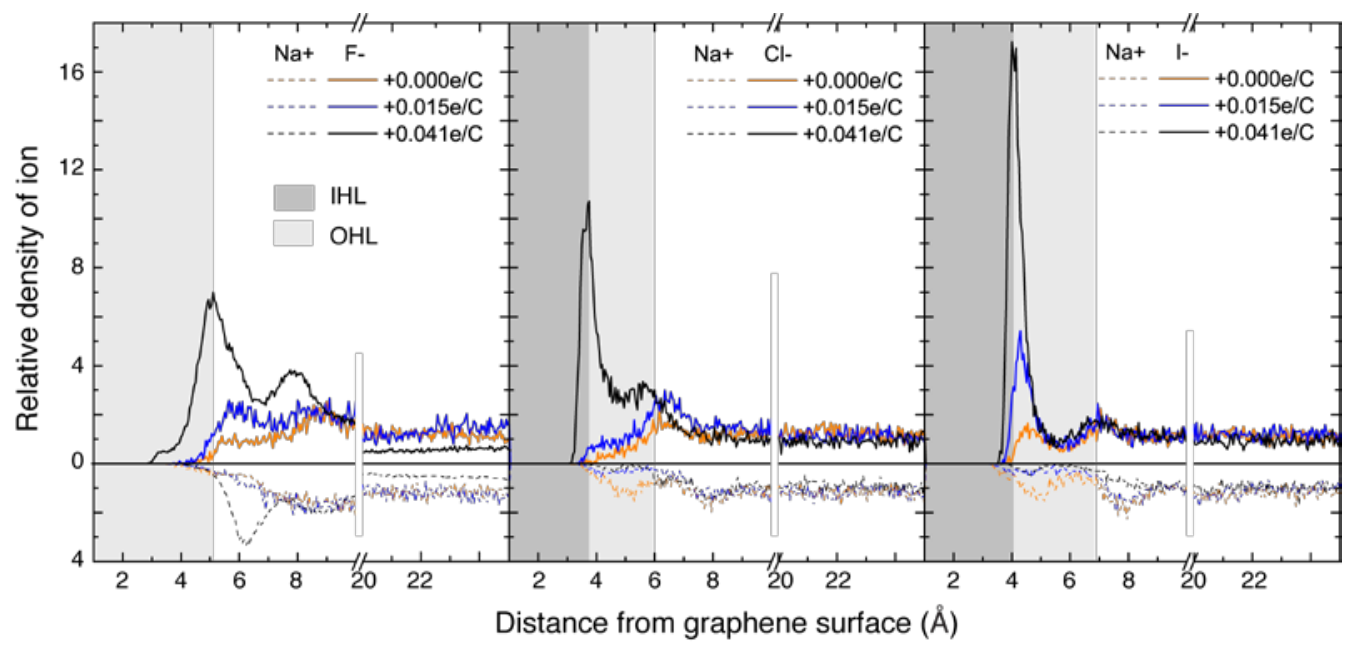


Figure 3. Relative number density $\rho^{*}$ of different electrolyte ions as a function of distance from a graphene anode surface with a charge of $0,+0.015$ and +0.041 e/C-atom, respectively. The shaded regions represent the IHL and OHL.

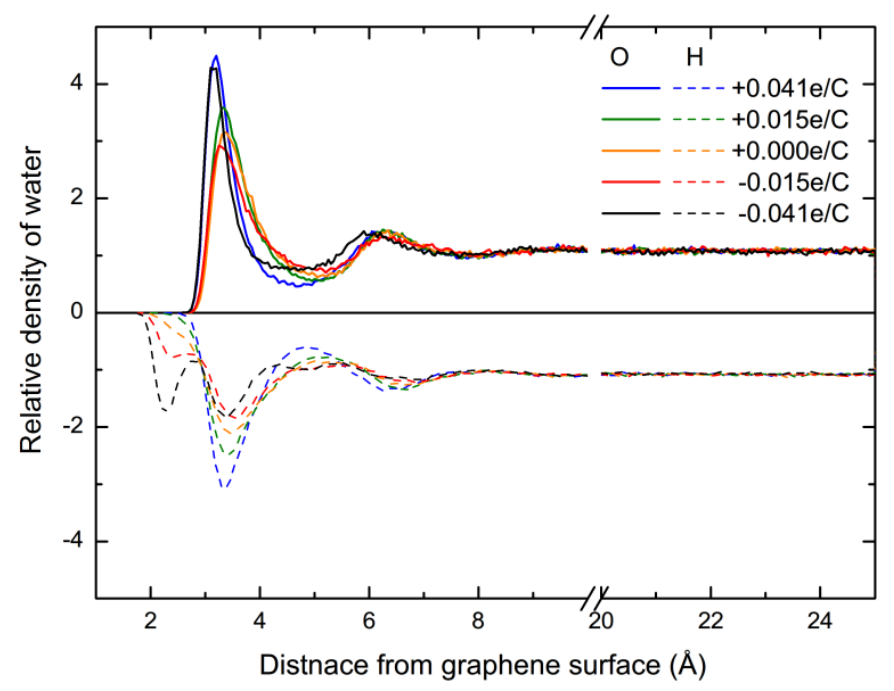

Figure 4. Relative number density $\rho^{*}$ of water molecules as a function of distance from a graphene anode surface with a charge of $+0.041,+0.015,0,-0.015$ and $-0.041 \mathrm{e} / \mathrm{C}$-atom, respectively. A negligible change of water distribution is seen in different electrolyte. The presented results are for the $\mathrm{NaCl}$ electrolyte.

The sharp relative number density peaks in Figs. 2 and 3 represent the accumulation of electrolyte ions as a result of the electrical interactions from the charged electrode surfaces. The regions between the peaks and the electrode surface are regarded as the impact Helmholtz layers (HLs) in the Grahame model. The ionic density gradually decays as the distance increases from the electrode surface, and ultimately to the nominal density in the bulk solution, the region of which is known as the diffusion layer. As the electrolyte in our MD simulations has a relatively high concentration of 1 M, the EDL is fairly compact and has a thickness of $\sim 1.0 \mathrm{~nm}$ (Figs. 2 and 3). This is generally consistent with the classic GCS model [44, 45]. Nevertheless, Figs. 2 and 3 clearly show an oscillating $\rho^{*}$, in contrast to the continuous exponential decay ionic density stated in the continuum GCS model. We found that the first electrolyte ion layer next to the graphene surface contains more opposite charges than the total charge of graphene electrode. As such, a following ion layer with an opposite charge is needed to counterbalance the surplus charges. The finite size of water molecules and ions in MD simulations has resulted in this over-screening effect [6, 26, 27, 46-48], which has been identified to negatively contribute to capacitance since it effectively increases the distance between electrode and counter-ions [49-52]. 
Figure 4 shows a clear water layer adsorbed on the graphene surface. The relative number density $\rho^{*}$ of $\mathrm{O}$ atom is estimated between 3 and 4 depending on the electrode surface charges (or the resultant electric field). It is often called as "the first water layer" in literatures [42, 53, 54]. The formation of this layer can be attributed to the interruption of continuous hydrogen-bonding network by the hydrophobic graphene surface. Without electrode surface charge, the $\rho^{*}$ of oxygen is about 3 and the distance to the electrode surface is about $3.2 \AA$ (Figs. 2 and 3), in good agreement with previous MD results [6, 26, 27, 55]. In the first water layer, the density profiles of hydrogen atoms are slightly closer to the graphene surface than those of oxygen atoms, indicating the water molecules having one of its $\mathrm{OH}$ bond pointing toward the graphene surface. It is worth noting that we did not see noticeable influences of the different electrolyte ions on the number density of the first water layer. Thus we only show results of electrolyte with $\mathrm{Na}^{+}$and $\mathrm{Cl}^{-}$solute ions in Fig. 4 .

Figure 2 shows clear structural differences of EDL for different electrolyte cations. The first water layer hinders the hydrated cations from moving towards the charged graphene surface [3, 56], resulting in the first density peaks of $\mathrm{Na}^{+}$and $\mathrm{K}^{+}$located behind the first water layer. Such a concentrated hydrated cation layer is known as the outer Helmholtz layer $(\mathrm{OHL})[18,57,58]$. The EDL structures of $\mathrm{Rb}^{+}$and $\mathrm{Cs}^{+}$cations are very different. For a surface charge of graphene cathode of $-0.015 \mathrm{e} / \mathrm{C}$-atom and above, a new ionic density peak starts to appear next to the electrode surface, overlapping with the first water layer. This new ionic density peak corresponds to the inner-Helmholtz layer (IHL) [57, 58], which arises from the distortion of the weak hydration shell of large-sized ions when approaching to an electrode surface under a strong external electrical field [25, 59-63].

Figure 3 summarizes the different EDL structures for the electrolyte anions close to the graphene anodes. The $\mathrm{F}^{-}$anion only forms $\mathrm{OHL}$, which is similar to $\mathrm{Na}^{+}$and $\mathrm{K}^{+}$but with a much larger distance from electrode surface. For the $\mathrm{Cl}^{-}$anion, only an OHL forms under a low surface charge of $+0.015 \mathrm{e} / \mathrm{C}$-atom. The result is in contrast to the previous report of specific absorption of $\mathrm{Cl}^{-}$on metal surface [64]. But an IHL emerges under a relatively high surface charge of $+0.041 \mathrm{e} / \mathrm{C}$-atom. The $\mathrm{I}^{-}$anion, even under a zero electrode surface charge, has a clear IHL ionic density peak shown in Fig. 3. This should be attributed to a strong affinity of $\mathrm{I}^{-}$to graphene, arising from its pronounced lone-pair electron densities [64]. Similar ions/electrode affinity has been discovered in the ionic-liquid/graphene system, leading to peculiar ionic distribution and orientation in the EDL [14, $16,65,66]$.

Some quantitative information of EDL structures for different electronic ions, such as the thickness of the IHL and OHL and the relative number density $\rho^{*}$, are summarized in Figs. 5 and 6. Overall, 
the thickness of the IHL/OHL increases with the cation/anion size, which is easy to understand. But in terms of the $\rho^{*}$ magnitude of OHLs, there is no clear trend on the ionic size in the case of 0.015 $\pm \mathrm{e} / \mathrm{C}$-atom electrode surface charge. The $\mathrm{K}^{+}$ion has the largest $\rho^{*}$ value of OHL among the cations and the $\mathrm{Cl}^{-}$has the largest $\rho^{*}$ value of $\mathrm{OHL}$ among the anions. Under a higher graphene surface charge, i.e., $0.041 \pm \mathrm{e} / \mathrm{C}$-atom, the $\rho^{*}$ of $\mathrm{OHL}$ exhibits a clear trend: decreasing with the ionic size. Note that the $\rho^{*}$ of IHL shows an opposite trend: increasing with the increase of the ion size. This is because it is relative easier for larger ions to dehydrate, leading to a more pronounced accumulation near the charged electrode surface (i.e., IHL). This effect partly contributes to the reduction of $\rho^{*}$ of OHL.

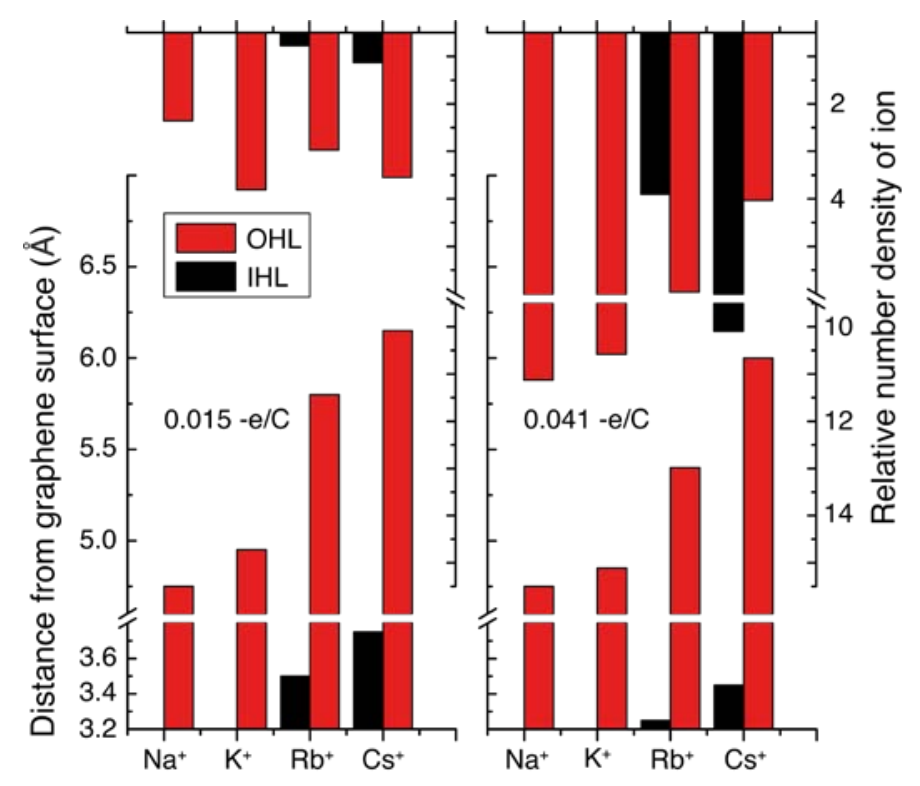

Figure 5. Thickness of the IHL and OHL at the graphene cathode surface and the relative ionic number density in the cases of electrode surface charge of $-0.015 \mathrm{e} / \mathrm{C}$-atom or $-0.041 \mathrm{e} / \mathrm{C}$-atom. 


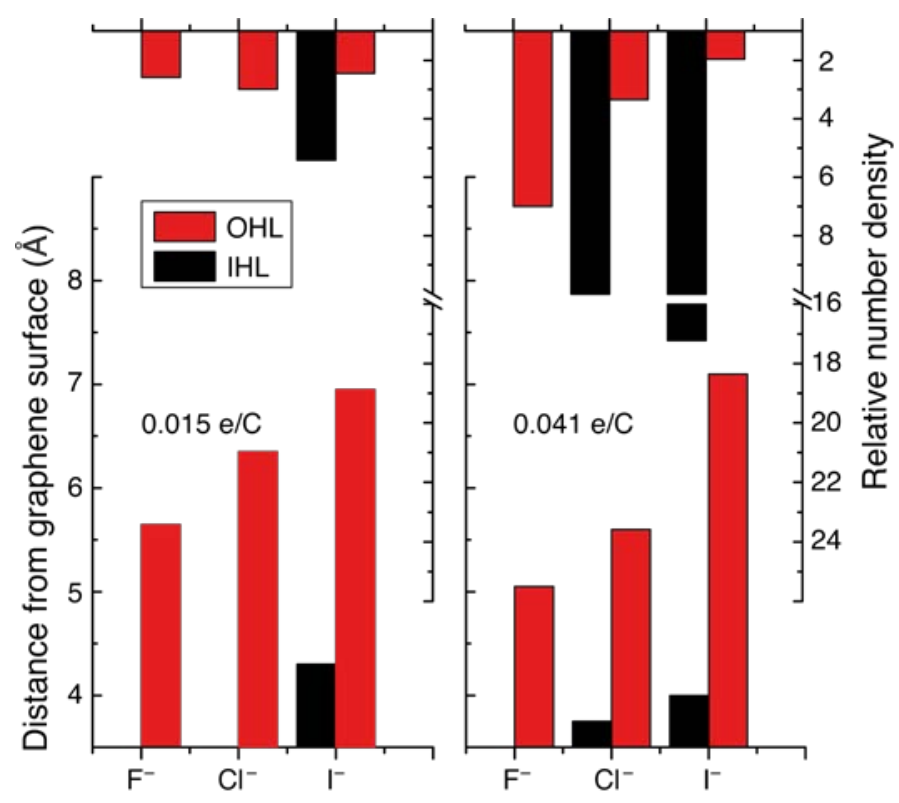

Figure 6. Thickness of the IHL and OHL at the graphene anode surface and relative ionic number density in the cases of electrode surface charge of $+0.015 \mathrm{e} / \mathrm{C}$-atom or $+0.041 \mathrm{e} / \mathrm{C}$-atom.

The different graphene surface charges significantly change the EDL structures as well as the first water layers. Generally, a larger surface charge leads to a reduction of the IHL/OHL thickness (i.e., ions moving toward the charged graphene surface) and an enhancement of the relative number density of the IHL/OHL layers (Figs. 5 and 6). There are two exceptions. There is nearly no change of OHL thickness for $\mathrm{Na}^{+}$cation. This may be due to the strong hydration shell of $\mathrm{Na}^{+}$. For the $\mathrm{I}^{-}$ anion, the OHL thickness increases with an increase of electrode surface charge. The formation of stronger IHL could push the OHL further away. Note that the enhancement of the $\rho^{*}$ of OHL and IHL is not proportional to the electrode surface charge change, e.g., 5 times increase of $\rho^{*}$ of the $\mathrm{Na}^{+}$OHL vs. a 2.67 times increase of electrode surface charges.

The electrode surface charge also alters the molecular structure of the first water layer, mainly manifested as the partial re-orientation of the water dipoles. The negative charge on the graphene cathode leads to the water dipole pointing toward the graphene surface, leading to the enhancement of hydrogen atom density (in front of the oxygen atom density peak) in Fig. 4. The positive charge of graphene anode yields an opposite effect. The changes in the water molecular network are expected to alter the dielectric of aqueous solution, and hence the capacitance values. This effect will be discussed in next section.

In a short summary, different electrolyte ions have profound effects on the EDL structures. The large size ions and those with specific affinity to the graphene could form IHL next to the charged electrode surface. The thickness of IHL and OHL increases with the ionic size. But the ionic density 
of IHL and OHL has no clear trend under a relative low surface charge (i.e., 0.015e/C-atom). An increase in surface charge generally causes a reduction of the thickness (except $\mathrm{Na}^{+}$and $\mathrm{I}^{-}$) and enhancement of the ionic density of the IHL and OHL (except $\mathrm{I}^{-}$). Note that different types of electrolyte ions have negligible effect on the water density profile. But different surface charge status has clear influences on water molecule re-orientation in the first water layer. The EDL structure and water solvent will determine the electric potential distribution and thus the capacitance results, which will be discussed in next section.

\subsection{Electric potential in the EDLs}

Based on the EDL structures obtained from MD simulations, this section analyzes the electric potential results and calculates the capacitance values of the graphene-based supercapacitors using different aqueous electrolytes and under different electrode surface charging states. The theoretical results are compared with our experimental results. The influences of ionic EDL structures and dielectrics of $\mathrm{H}_{2} \mathrm{O}$ solvent on the capacitance are carefully examined.

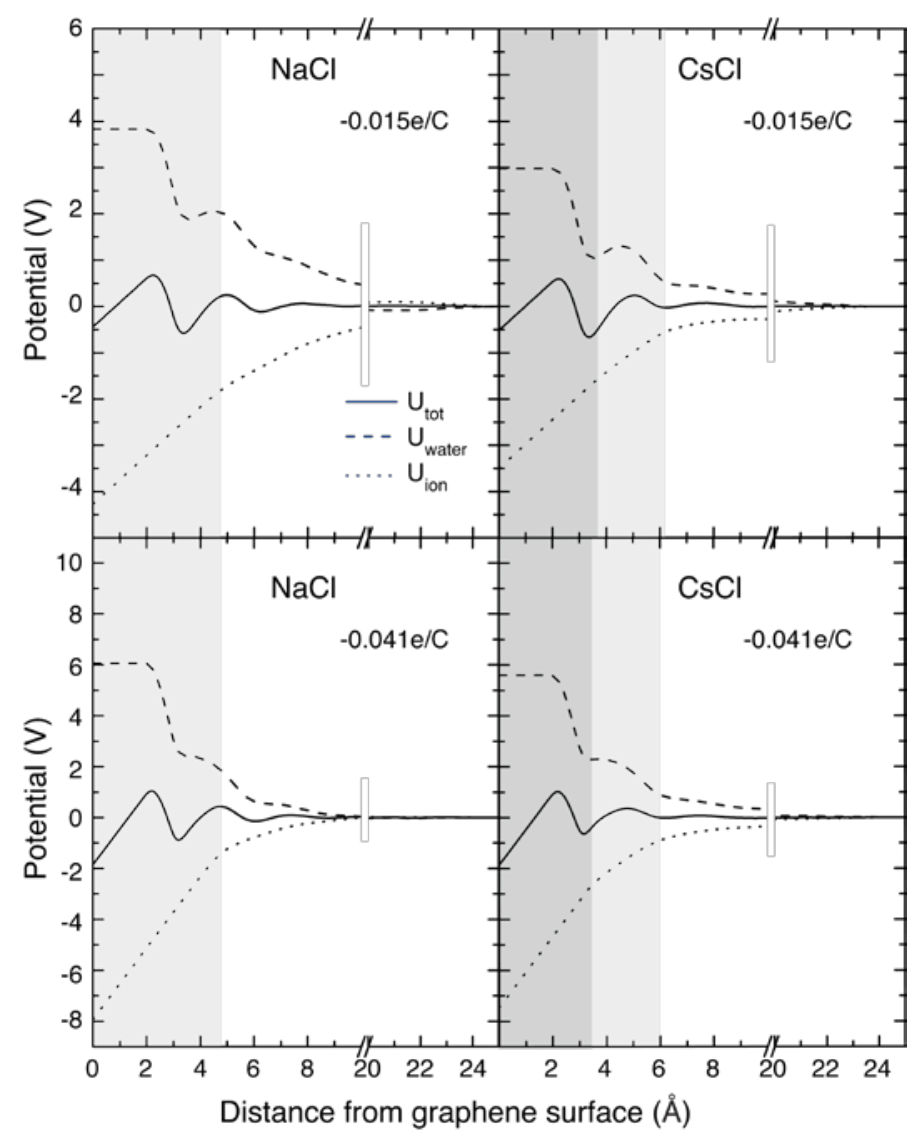


Figure 7. The electric potential $V$ as a function of distance $x$ from the graphene cathode surface for the $\mathrm{NaCl}$ and $\mathrm{CsCl}$ electrolyte under two charging cases: $-0.015 \mathrm{e} / \mathrm{C}$-atom and -0.041e/C-atom. The total electrical potential $U_{\text {tot }}$ is decomposed into two components: $U_{\text {ion }}$ calculated from the ion EDL $\rho(x)$ and the cathode surface charge, and $U_{\text {water }}$ calculated from the water molecular dipoles.

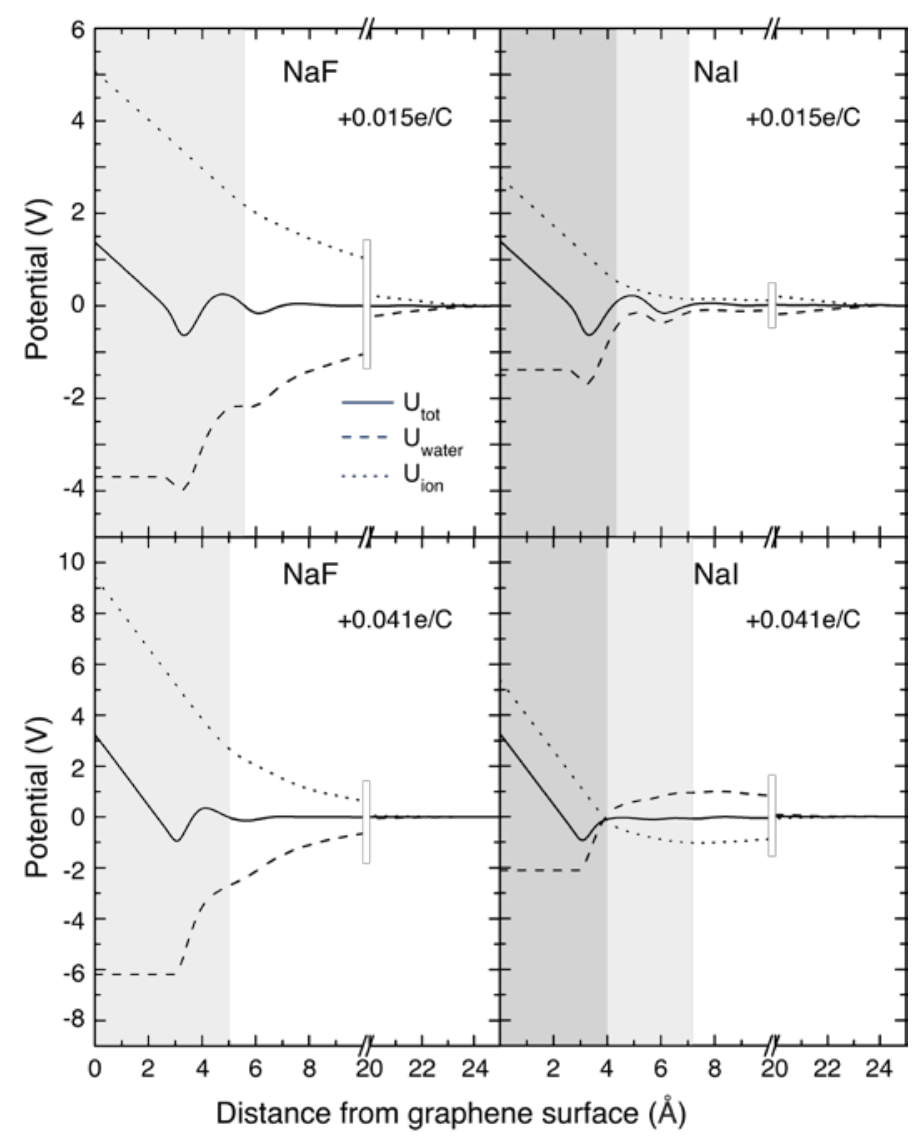

Figure 8. The electric potential $V$ as a function of distance $x$ from the graphene cathode surface for the NaF and NaI electrolyte under two charging cases: $+0.015 \mathrm{e} / \mathrm{C}$-atom and $+0.041 \mathrm{e} / \mathrm{C}$-atom. The total electrical potential $U_{\text {tot }}$ is decomposed into two components: $U_{\text {ion }}$ calculated from the ion EDL $\rho(x)$ and the anode surface charge, and $U_{\text {water }}$ calculated from the water molecular dipoles.

Figure 7 shows the calculated electric potentials $V(x)$ for the $\mathrm{NaCl}$ and $\mathrm{CsCl}$ electrolyte under two charging cases: a surface charge of $-0.015 \mathrm{e} / \mathrm{C}$-atom or $-0.041 \mathrm{e} / \mathrm{C}$-atom, at a graphene cathode. Figure 8 summarizes the results for $\mathrm{NaF}$ and $\mathrm{NaI}$ electrolytes next to a graphene anode. To clearly examine the roles of electrolyte ions and water solvent on capacitance results, the electric potentials $U_{\text {tot }}$ were decomposed into contributions from the ionic EDL $\left(U_{\text {ion }}\right)$ and the water solvent $\left(U_{\text {water }}\right)$ separately. The $U_{\text {ion }}$ is computed based on ionic EDL $\rho(x)$ and the electrode surface charge. The $U_{\text {water }}$ is computed by only considering the polarized water molecules. 
With the obtained $U_{\text {tot, }}$ we calculated the capacitance value and summarized the results in Table 3 and 4. First, the calculated capacitance values in our simulations (100 280 F/g) are quite close to the experiment results $(130 \sim 300 \mathrm{~F} / \mathrm{g})$ [39, 67, 68]. Our experimental results have indicated that the capacitance of cathode would be $\sim 100 \mathrm{~F} / \mathrm{g}$ higher than that of anode, which reasonably agrees with our simulation results (see supporting information). Second, increasing the cathode surface charge density leads to significantly reduced specific capacitance values (Table 3). But such a reduction is not observed for graphene anodes. Our experiments appear to show a more significant change of capacitance values for cathodes when varying from $-0.25 \mathrm{~V}$ and $-0.4 \mathrm{~V}$ than that of the anodes, i.e., $5.75 \mathrm{~F} / \mathrm{g}$ vs. $0.83 \mathrm{~F} / \mathrm{g}$ (see supporting information), which is consistent with our MD results. But magnitude of the changes in MD simulations (Table 3) is much higher. This could be partly attributed to the much higher voltage in our MD simulations (Table 3). Third, it is a surprise to notice that the specific capacitance values of the cathodes and anodes weakly depend on the electrolytes ions types, despite the significantly different EDL structures as observed in Figs. 2 and 3. This is supported by our experimental results that capacitance values of graphene cathode using the $\mathrm{LiCl}, \mathrm{NaCl}, \mathrm{KCl}$, and $\mathrm{CsCl}$ electrolytes are all around 240F/g [69]. In the following, we will provide an in-depth analysis to understand these observations.

Table 3. Specific capacitance of a graphene cathode immersed in different electrolytes, with a surface charge of $-0.015 \mathrm{e} / \mathrm{C}$-atom or $-0.041 \mathrm{e} / \mathrm{C}$-atom.

\begin{tabular}{ccc}
\hline (F/g) & $-0.015 \mathrm{e} / \mathrm{C}-$ atom & $-0.041 \mathrm{e} / \mathrm{C}$-atom \\
\hline $\mathrm{NaCl}$ & 279.8 & 176.4 \\
$\mathrm{KCl}$ & 282.7 & 175.5 \\
$\mathrm{RbCl}$ & 254.9 & 178.2 \\
$\mathrm{CsCl}$ & 237.9 & 174.5 \\
\hline
\end{tabular}

Table 4. Specific capacitance of a graphene anode immersed in different electrolyte, with a surface charge of 0.015e/C-atom and 0.041e/C-atom.

\begin{tabular}{ccc}
\hline$(\mathrm{F} / \mathrm{g})$ & $+0.015 \mathrm{e} / \mathrm{C}-$ atom & $+0.041 \mathrm{e} / \mathrm{C}-$ atom \\
\hline $\mathrm{NaF}$ & 89.14 & 99.47 \\
$\mathrm{NaCl}$ & 90.53 & 100.9 \\
$\mathrm{NaI}$ & 87.70 & 99.66 \\
\hline
\end{tabular}


The $U_{\text {ion }}$ results of different electrolyte ions are significantly different (Figs. 7 and 8). For the $U_{\text {ion, a }}$ linear relation is observed in the EDL region 4-6 $\AA$ from graphene surface. This region corresponds to the OHL/IHL in Fig. 2. It can be treated as a parallel plate capacitor composed of the Holmholtz layer and the charged electrode, manifested by the linear electric potential. In the diffusive layer, $U_{\text {ion }}$ gradually approaches the reference state (i.e., zero in bulk electrolyte). The $\mathrm{NaCl}$ has larger $U_{\text {ion }}$ values at the graphene electrode than the $\mathrm{CsCl}$ under both charging cases. This can be understood by the formation of IHL in the CsCl case (Fig. 2), which effectively reduces the thickness of EDL. For the same reason (Fig. 3), the NaI has a smaller $U_{\text {ion }}$ value at electrode surface than NaF (Fig. 8).

The $U_{\text {water }}$ exhibits a significant oscillation. An electric potential plateau is observed in the water depletion region (between the graphene and the first water layer). The dipoles caused by the reorientation of water molecules being subjected to external electric field (Fig. 4) result in the sharp potential drop in $U_{\text {water }}$ from 2 to $3 \AA$ (Figs. 7 and 8). The $U_{\text {water }}$ has an opposite sign to $U_{\text {ion. }}$ Additionally, a higher $U_{\text {ion }}$ value is always accompanied by a higher $U_{\text {water }}$ value for different electrolyte cases. Both facts reflect the dielectric nature of water solvent.

Despite the distinct EDL structures and the $U_{\text {ion }}$ results of $\mathrm{NaCl}, \mathrm{KCl}, \mathrm{RuCl}$, and $\mathrm{CsCl}$ aqueous electrolytes (Figs. 2 and 7), their total electric potential profiles $U_{\text {tot }}$ are quite similar, resulting in a similar capacitance value (Table 2 and 5). Similar situation is observed for the graphene anode cases (Fig. 8 and Table 3 and 6). It is clear that the dielectric water molecules play a critical role in determining the overall potential drop in EDLs [70, 71].

The critical role of water solvent in determining the capacitance values of different electrolyte can be understood in terms of the dielectric constants in the EDL ( $\varepsilon_{\text {water }}$ ), as summarized in Table 5, Table 6, and Table S2. We found a general trend that a larger ion tends to have a lower $\varepsilon_{\text {water: }} 9.744$ $\left(\mathrm{Na}^{+}\right)>7.266\left(\mathrm{Rb}^{+}\right)>6.788\left(\mathrm{Cs}^{+}\right)$and $3.693\left(\mathrm{~F}^{-}\right)>2.151\left(\mathrm{Cl}^{-}\right)>1.991\left(\mathrm{I}^{-}\right)$. It is such a reduction in dielectric constant that largely cancels out the beneficial effect on capacitance from a thinner EDL (thus a small $U_{\text {ion}}$ ) for those large ions $\mathrm{Rb}^{+}, \mathrm{Cs}^{+}, \mathrm{Cl}^{-}$, and $\mathrm{I}^{-}$, which results in small differences in the calculated capacitance values of different electrolyte ions. Note that recently a similar phenomenon was reported in supercapacitors using the organic ionic liquid (IL) electrolyte, in which the beneficial nanofinement effect for the ILs was cancelled by the reduction of the dielectric constant of the organic solvent [72]. Thus, variation of the dielectric constant of water or other solvent in the EDLs should be taken into account for the design of supercapacitors. 
Table 5. The electric potential drop (in unit of V) next to a graphene cathode with a charge of 0.015e/C-atom or $-0.041 \mathrm{e} / \mathrm{C}$-atom.

\begin{tabular}{ccccccccc}
\hline & \multicolumn{2}{c}{$\mathrm{NaCl}$} & \multicolumn{2}{c}{$\mathrm{KCl}$} & \multicolumn{2}{c}{$\mathrm{RuCl}$} & \multicolumn{2}{c}{$\mathrm{CsCl}$} \\
\hline $\begin{array}{c}\text { Surface charge } \\
(\mathrm{e} / \mathrm{C} \text {-atom })\end{array}$ & +0.015 & +0.041 & +0.015 & +0.041 & +0.015 & +0.041 & +0.015 & +0.041 \\
\hline$U_{\text {ion }}$ & 4.267 & 7.918 & 2.341 & 7.533 & 3.492 & 7.621 & 3.496 & 7.469 \\
$U_{\text {water }}$ & -3.829 & -6.066 & -1.907 & -5.671 & -3.011 & -5.787 & -2.981 & -5.598 \\
$U_{\text {tot }}$ & 0.4379 & 1.852 & 0.4334 & 1.862 & 0.4806 & 1.834 & 0.5150 & 1.872 \\
\hline$\varepsilon_{\text {water }}$ & 9.744 & 4.275 & 5.401 & 4.046 & 7.266 & 4.155 & 6.788 & 3.990 \\
\hline
\end{tabular}

Table 6. The electric potential drop (in unit of V) next to a graphene anode with a charge of $+0.015 \mathrm{e} / \mathrm{C}$-atom or $+0.041 \mathrm{e} / \mathrm{C}$-atom.

\begin{tabular}{ccccccc}
\hline & \multicolumn{2}{c}{$\mathrm{NaF}$} & \multicolumn{2}{c}{$\mathrm{NaCl}$} & \multicolumn{2}{c}{$\mathrm{NaI}$} \\
\hline $\begin{array}{c}\text { Surface charge } \\
(\mathrm{e} / \mathrm{C}-\text { atom })\end{array}$ & +0.015 & +0.041 & +0.015 & +0.041 & +0.015 & +0.041 \\
\hline$U_{\text {ion }}$ & -5.074 & -9.526 & -2.910 & -6.462 & -2.781 & -5.385 \\
$U_{\text {water }}$ & 3.700 & 6.241 & 1.556 & 3.223 & 1.383 & 2.106 \\
$U_{\text {tot }}$ & -1.374 & -3.285 & -1.353 & -3.239 & -1.397 & -3.278 \\
\hline$\varepsilon_{\text {EDL }}$ & 3.693 & 2.900 & 2.151 & 1.995 & 1.991 & 1.643 \\
\hline
\end{tabular}

The water solvent also plays an important role in determining the capacitance values at graphene cathodes and anodes. From Table 5 and $6, \mathrm{NaCl}$ has a weaker $U_{\text {ion }}$ than that of graphene cathode, yet the $U_{\text {tot }}$ shows an opposite trend. This is because the calculated $\varepsilon_{\text {water }}$ values in graphene anode side (Table 6) are significantly lower than the results for that in cathode (Table 5). This might be understood in terms of the re-orientation of the water molecules in the first water layer under different electrical fields. As shown in Fig. 4, the water molecules in the first water layer naturally have one of its $\mathrm{O}-\mathrm{H}$ bonds pointing toward the graphene surface (with zero charge). At the anode surface, the positive charge has to overcome such a tendency to re-orientate the $\mathrm{OH}$ bond pointing away from the anode surface. In other words, the water molecules near the anode surface are relatively more difficult to be polarized and thus the dielectric constant is smaller. In the end, the calculated capacitance values of graphene anodes are about 100F/g lower than that of cathodes, which agrees with our experimental observation quite well (see supporting information). 
The surface charging conditions also affect the overall capacitance values. First, a higher density of electrode surface charges will enhance the attraction of counter-ions towards graphene electrode and thus reduce the thickness of EDLs (Fig. 4 and 5). The increase of $U_{\text {ion }}$ is less proportional to the increase of surface charge, suggesting a tendency of capacitance enhancement. But when subjected to a high electric field, the water molecules are highly orientated (or ordered) thus have less capability to re-orientated further. The dielectric constants are smaller than those in the weak electric field (the so-called “dielectric saturation”) [44, 73, 74], which can be seen in our calculated results in Table 5 and 6. Overall, for graphene cathode, the dielectric saturation effect dominates at strong surface charging. We, therefore, obtain significantly reduced capacitances, i.e., about 30-40\% reduction (Table 3). But for anodes, these two contributions almost cancel out each other. There is, therefore, a negligible change in capacitance (Table 4).

\section{Conclusion}

In summary, $\mathrm{MD}$ simulations were carried out for a comparative study on the EDL molecular structures of $\mathrm{M}^{+} \mathrm{X}^{-}\left(\mathrm{M}^{+}=\mathrm{Na}^{+}, \mathrm{K}^{+}, \mathrm{Ru}^{+}\right.$, and $\mathrm{Cs}^{+} ; \mathrm{X}^{-}=\mathrm{F}^{-}, \mathrm{Cl}^{-}$, and $\left.\mathrm{I}^{-}\right)$electrolytes at the graphene cathodes and anodes, under different charging conditions. The role of electrolyte ion types, water solvent, and the surface charging condition on the total capacitance values are investigated. We found that the larger ions, such as $\mathrm{Rb}^{+}, \mathrm{Cs}^{+}, \mathrm{Cl}^{-}$and $\mathrm{I}^{-}$, will be dehydrated subject to the external electric field (with certain strength) and penetrate the first water layer to form the impact Helmholtz layer (IHL). Generally, thickness of the IHL and OHL layer increases with the size of ions. But there is no clear trend for the ionic number density of the OHL/IHL with respect to the ionic size. The formation of IHL will effectively reduce the thickness of the whole EDL, leading to a smaller electric potential drop $U_{\text {ion }}$ for the large ions under the same electrode charging condition. Our results reveal the critical role of water solvent in determining the capacitance values. First, despite the significant difference observed in EDL structure and the $U_{\text {ion }}$ values for different electrolyte, the changes of water dielectric constants cancel out the influences, leading to a nearly the same capacitance value, which agrees with our experiments. Second, the electric potential drop $U_{\text {ion }}$ from the EDL of graphene anodes is smaller than that of the graphene cathodes, potentially could lead to a higher capacitance value. Nevertheless, the water orientation at graphene anode surface results in a much smaller dielectric constant, and the graphene cathodes actually have a much higher capacitance than anodes. This is in a good agreement with our experimental results. Third, we notice that a higher surface charging condition will reduce the water dielectric constants and thus cause a significant drop of capacitance, which is also discovered within the experiments. 


\section{Acknowledgement}

The authors acknowledge the support of discovery projects from Australian Research Council and National Computational Infrastructure at Australian National University.

\section{References}

1. Chmiola, J., et al., Anomalous Increase in Carbon Capacitance at Pore Sizes Less Than 1 Nanometer. Science, 2006. 313(5794): p. 1760-1763.

2. Huang, J., B.G. Sumpter, and V. Meunier, A Universal Model for Nanoporous Carbon Supercapacitors Applicable to Diverse Pore Regimes, Carbon Materials, and Electrolytes. Chemistry - A European Journal, 2008. 14(22): p. 6614-6626.

3. Cagle, C., et al., Structure and charging kinetics of electrical double layers at large electrode voltages. Microfluidics and Nanofluidics, 2010. 8(5): p. 703-708.

4. Feng, G., et al., Atomistic Insight on the Charging Energetics in Subnanometer Pore Supercapacitors. The Journal of Physical Chemistry C, 2010. 114(41): p. 18012-18016.

5. Jiang, D.-e., Z. Jin, and J. Wu, Oscillation of Capacitance inside Nanopores. Nano Letters, 2011. 11(12): p. 5373-5377.

6. Wander, M.C.F. and K.L. Shuford, Electrolyte Effects in a Model System for Mesoporous Carbon Electrodes. The Journal of Physical Chemistry C, 2011. 115(11): p. 4904-4908.

7. Górniak, R. and S. Lamperski, Investigation of the Electrical Double Layer with a Graphene Electrode by the Grand Canonical Monte Carlo Simulation. The Journal of Physical Chemistry C, 2014. 118(6): p. 3156-3161.

8. Punnathanam, S.N., A Gibbs-ensemble based technique for Monte Carlo simulation of electric double layer capacitors (EDLC) at constant voltage. The Journal of Chemical Physics, 2014. 140(17): p. 174110-9.

9. Jiang, D.-e. and J. Wu, Microscopic Insights into the Electrochemical Behavior of Nonaqueous Electrolytes in Electric Double-Layer Capacitors. The Journal of Physical Chemistry Letters, 2013. 4(8): p. 1260-1267.

10. DeYoung, A.D., et al., Graphene Oxide Supercapacitors: A Computer Simulation Study. The Journal of Physical Chemistry C, 2014. 118(32): p. 18472-18480.

11. Feng, G., et al., Molecular Insights into Carbon Supercapacitors Based on Room-Temperature lonic Liquids. The Journal of Physical Chemistry Letters, 2013. 4(19): p. 3367-3376.

12. Kondrat, S., et al., A superionic state in nano-porous double-layer capacitors: insights from Monte Carlo simulations. Physical Chemistry Chemical Physics, 2011. 13(23): p. 11359-11366.

13. Kondrat, S. and A. Kornyshev, Superionic state in double-layer capacitors with nanoporous electrodes. Journal of Physics: Condensed Matter, 2011. 23(2): p. 022201-5.

14. Shim, Y., Y. Jung, and H.J. Kim, Graphene-Based Supercapacitors: A Computer Simulation Study. The Journal of Physical Chemistry C, 2011. 115(47): p. 23574-23583.

15. Vatamanu, J., O. Borodin, and G.D. Smith, Molecular Insights into the Potential and Temperature Dependences of the Differential Capacitance of a Room-Temperature lonic Liquid at Graphite Electrodes. Journal of the American Chemical Society, 2010. 132(42): p. 14825-14833.

16. Vatamanu, J., O. Borodin, and G.D. Smith, Molecular Dynamics Simulation Studies of the Structure of a Mixed Carbonate/LiPF6 Electrolyte near Graphite Surface as a Function of Electrode Potential. The Journal of Physical Chemistry C, 2011. 116(1): p. 1114-1121.

17. Vatamanu, J., et al., On the Influence of Surface Topography on the Electric Double Layer Structure and Differential Capacitance of Graphite/Ionic Liquid Interfaces. The Journal of Physical Chemistry Letters, 2011. 2(17): p. 2267-2272. 
18. Gouy, G., Constitution of the electric charge at the surface of an electrolyte. J. phys, 1910. 9(4): p. 457-467.

19. Helmholtz, H.v., Ueber einige Gesetze der Vertheilung elektrischer Ströme in körperlichen Leitern mit Anwendung auf die thierisch - elektrischen Versuche. Annalen der Physik, 1853. 165(6): p. 211-233.

20. Stern-Hamburg, H.O., ZUR THEORIE- DER ELEKTROLYTISCHEN DOPPELSCHICHT. S. f. Electrochemie, 1924. 30: p. 508-516.

21. Chapman, D.L., LI. A contribution to the theory of electrocapillarity. The London, Edinburgh, and Dublin Philosophical Magazine and Journal of Science, 1913. 25(148): p. 475-481.

22. Xia, J., et al., Measurement of the quantum capacitance of graphene. Nat Nano, 2009. 4(8): p. 505-509.

23. Feng, G. and P.T. Cummings, Supercapacitor Capacitance Exhibits Oscillatory Behavior as a Function of Nanopore Size. The Journal of Physical Chemistry Letters, 2011. 2(22): p. 2859-2864.

24. Feng, G., D.-e. Jiang, and P.T. Cummings, Curvature Effect on the Capacitance of Electric Double Layers at Ionic Liquid/Onion-Like Carbon Interfaces. Journal of Chemical Theory and Computation, 2012. 8(3): p. 1058-1063.

25. Kalluri, R.K., et al., Partition and Structure of Aqueous NaCl and $\mathrm{CaCl} 2$ Electrolytes in Carbon-Slit Electrodes. The Journal of Physical Chemistry C, 2013. 117(26): p. 13609-13619.

26. Wander, M.C.F. and K.L. Shuford, Molecular Dynamics Study of Interfacial Confinement Effects of Aqueous NaCl Brines in Nanoporous Carbont. The Journal of Physical Chemistry C, 2010. 114(48): p. 20539-20546.

27. Wander, M.C.F. and K.L. Shuford, Alkali Halide Interfacial Behavior in a Sequence of Charged Slit Pores. The Journal of Physical Chemistry C, 2011. 115(47): p. 23610-23619.

28. Reed, S.K., O.J. Lanning, and P.A. Madden, Electrochemical interface between an ionic liquid and a model metallic electrode. The Journal of Chemical Physics, 2007. 126(8): p. 84704-84716.

29. Péan, C., et al., On the Dynamics of Charging in Nanoporous Carbon-Based Supercapacitors. ACS Nano, 2014. 8(2): p. 1576-1583.

30. Merlet, C., et al., Simulating Supercapacitors: Can We Model Electrodes As Constant Charge Surfaces? The Journal of Physical Chemistry Letters, 2012. 4(2): p. 264-268.

31. Wang, Z., et al., Evaluation of the constant potential method in simulating electric double-layer capacitors. The Journal of Chemical Physics, 2014. 141(18): p. 184102-184107.

32. Jorgensen, W.L., Quantum and statistical mechanical studies of liquids. 10. Transferable intermolecular potential functions for water, alcohols, and ethers. Application to liquid water. Journal of the American Chemical Society, 1981. 103(2): p. 335-340.

33. Ryckaert, J.-P., G. Ciccotti, and H.J.C. Berendsen, Numerical integration of the cartesian equations of motion of a system with constraints: molecular dynamics of $n$-alkanes. Journal of Computational Physics, 1977. 23(3): p. 327-341.

34. MacKerell, A.D., et al., All-Atom Empirical Potential for Molecular Modeling and Dynamics Studies of Proteinst. The Journal of Physical Chemistry B, 1998. 102(18): p. 3586-3616.

35. Foloppe, N. and J.A.D. MacKerell, All-atom empirical force field for nucleic acids: I. Parameter optimization based on small molecule and condensed phase macromolecular target data. Journal of Computational Chemistry, 2000. 21(2): p. 86-104.

36. Dang, L.X. and B.C. Garrett, Photoelectron spectra of the hydrated iodine anion from molecular dynamics simulations. The Journal of Chemical Physics, 1993. 99(4): p. 2972-2977.

37. Dang, L.X., Fluoride-fluoride association in water from molecular dynamics simulations. Chemical Physics Letters, 1992. 200(1-2): p. 21-25.

38. Fasolino, A., J.H. Los, and M.I. Katsnelson, Intrinsic ripples in graphene. Nat Mater, 2007. 6(11): p. 858-861.

39. Yang, X., et al., Bioinspired Effective Prevention of Restacking in Multilayered Graphene Films: Towards the Next Generation of High-Performance Supercapacitors. Advanced Materials, 2011. 23(25): p. 2833-2838.

40. Yang, X., et al., Liquid-Mediated Dense Integration of Graphene Materials for Compact Capacitive Energy Storage. Science, 2013. 341(6145): p. 534-537. 
41. Ma, M.D., et al., Friction of water slipping in carbon nanotubes. Physical Review E, 2011. 83(3): p. 036316-.

42. Xiong, W., et al., Strain engineering water transport in graphene nanochannels. Physical Review E, 2011. 84(5): p. 056329-7.

43. Chen, C., et al., Nanoscale fluid-structure interaction: Flow resistance and energy transfer between water and carbon nanotubes. Physical Review E, 2011. 84(4): p. 046314.

44. Conway, B., Electrochemical supercapacitors: scientific fundamentals and technological applications (POD). 1999: Kluwer Academic/Plenum: New York.

45. Behrens, S.H. and M. Borkovec, Exact Poisson-Boltzmann solution for the interaction of dissimilar charge-regulating surfaces. Physical Review E, 1999. 60(6): p. 7040-7048.

46. Wang, S., et al., Molecular Dynamic Simulations of Ionic Liquids at Graphite Surface. The Journal of Physical Chemistry C, 2009. 114(2): p. 990-995.

47. Spohr, E., Molecular simulation of the electrochemical double layer. Electrochimica Acta, 1999. 44(11): p. 1697-1705.

48. Crozier, P.S., R.L. Rowley, and D. Henderson, Molecular dynamics calculations of the electrochemical properties of electrolyte systems between charged electrodes. The Journal of Chemical Physics, 2000. 113(20): p. 9202-9207.

49. Bazant, M.Z., B.D. Storey, and A.A. Kornyshev, Double Layer in Ionic Liquids: Overscreening versus Crowding. Physical Review Letters, 2011. 106(4): p. 046102.

50. Fedorov, M.V. and A.A. Kornyshev, Towards understanding the structure and capacitance of electrical double layer in ionic liquids. Electrochimica Acta, 2008. 53(23): p. 6835-6840.

51. Merlet, C., et al., On the molecular origin of supercapacitance in nanoporous carbon electrodes. Nat Mater, 2012. 11(4): p. 306-310.

52. Feng, G., et al., A "counter-charge layer in generalized solvents" framework for electrical double layers in neat and hybrid ionic liquid electrolytes. Physical Chemistry Chemical Physics, 2011. 13(32): p. $14723-14734$.

53. Falk, K., et al., Molecular Origin of Fast Water Transport in Carbon Nanotube Membranes: Superlubricity versus Curvature Dependent Friction. Nano Letters, 2010. 10(10): p. 4067-4073.

54. Yang, K.-L., S. Yiacoumi, and C. Tsouris, Monte Carlo simulations of electrical double-layer formation in nanopores. The Journal of Chemical Physics, 2002. 117(18): p. 8499-8507.

55. Marti, J., et al., Molecular simulation of liquid water confined inside graphite channels: Thermodynamics and structural properties. The Journal of Chemical Physics, 2006. 124(9): p. -.

56. Cui, S.T. and H.D. Cochran, Molecular dynamics simulation of interfacial electrolyte behaviors in nanoscale cylindrical pores. The Journal of Chemical Physics, 2002. 117(12): p. 5850-5854.

57. Hunter, R.J., Zeta Potential in Colloid Science: Principles and Applications. 1988: Academic Press.

58. Schoch, R.B., H. van Lintel, and P. Renaud, Effect of the surface charge on ion transport through nanoslits. Physics of Fluids (1994-present), 2005. 17(10): p. -.

59. Mähler, J. and I. Persson, A Study of the Hydration of the Alkali Metal Ions in Aqueous Solution. Inorganic Chemistry, 2011. 51(1): p. 425-438.

60. Aaqvist, J., lon-water interaction potentials derived from free energy perturbation simulations. The Journal of Physical Chemistry, 1990. 94(21): p. 8021-8024.

61. Kalluri, R.K., et al., Unraveling the potential and pore-size dependent capacitance of slit-shaped graphitic carbon pores in aqueous electrolytes. Physical Chemistry Chemical Physics, 2013. 15(7): p. 2309-2320.

62. Rajamani, S., T. Ghosh, and S. Garde, Size dependent ion hydration, its asymmetry, and convergence to macroscopic behavior. The Journal of Chemical Physics, 2004. 120(9): p. 4457-4466.

63. Richens, D., The chemistry of aqua ions: synthesis, structure, and reactivity: a tour through the periodic table of the elements. J. 1997, Wiley, Chichester and New York.

64. Magnussen, O.M., Ordered Anion Adlayers on Metal Electrode Surfaces. Chemical Reviews, 2002. 102(3): p. 679-726.

65. Feng, G., et al., Structure and dynamics of electrical double layers in organic electrolytes. Physical Chemistry Chemical Physics, 2010. 12(20): p. 5468-5479. 
66. Singh, R., et al., Molecular dynamics simulations of the ionic liquid [EMIM+][TFMSI-] confined inside rutile (110) slit nanopores. Physical Chemistry Chemical Physics, 2013. 15(38): p. 16090-16103.

67. Stoller, M.D., et al., Graphene-Based Ultracapacitors. Nano Letters, 2008. 8(10): p. 3498-3502.

68. Zhang, Y., et al., Progress of electrochemical capacitor electrode materials: A review. International Journal of Hydrogen Energy, 2009. 34(11): p. 4889-4899.

69. Cheng, C., et al., Multilayered graphene membrane as an experimental platform to probe nano-confined electrosorption. Progress in Natural Science: Materials International, 2012. 22(6): p. 668-672.

70. Dimitrov, D.I., N.D. Raev, and K.I. Semerdzhiev, Molecular dynamics simulations of the electrical double layer at $1 \mathrm{M}$ potassium halide solution/Hg electrode interfaces. Physical Chemistry Chemical Physics, 2001. 3(3): p. 448-452.

71. Feng, G., et al., Ion Distribution in Electrified Micropores and Its Role in the Anomalous Enhancement of Capacitance. ACS Nano, 2010. 4(4): p. 2382-2390.

72. Kondrat, S., et al., The effect of dielectric permittivity on the capacitance of nanoporous electrodes. Electrochemistry Communications, 2013. 34: p. 348-350.

73. Yang, L., et al., Dielectric saturation of liquid propylene carbonate in electrical energy storage applications. The Journal of Chemical Physics, 2010. 132(4): p. 044701-4.

74. Fulton, R.L., The nonlinear dielectric behavior of water: Comparisons of various approaches to the nonlinear dielectric increment. The Journal of Chemical Physics, 2009. 130(20): p. 204503-204512.

Ref. A1: Alessio Alexiadis and Stavros Kassinos*, Molecular Simulation of Water in Carbon Nanotubes, Chem. Rev. 2008, 108, 5014-5034 